\title{
QUALIDADE MICROBIOLÓGICA DE COUVE MANTEIGA (BRASSICA OLERACEA) MINIMAMENTE PROCESSADA COMERCIALIZADA EM SÃO PAULO, BRASIL
}

\section{MICROBIOLOGICAL QUALITY OF MINIMALLY PROCESSED KALE (BRASSICA OLERACEA) SOLD IN SUPERMARKETS IN SÃO PAULO, BRAZIL}

\author{
Gracielle Gesteira Rocha ${ }^{1}$ \\ Alice Michi Chibana Miyagi' \\ Lívia Itapema Guimarães ${ }^{1}$ \\ Vanessa de Lima Cardoso ${ }^{1}$ \\ Andrea Carvalheiro Guerra Matias ${ }^{2}$ \\ Edeli Simione de Abreu²
}

Resumo: $O$ aumento da procura por alimentos prontos para consumo incentiva o desenvolvimento de novas tecnologias para o processamento de alimentos. Dentro desse contexto, alimentos frescos atraem os consumidores, promovendo a ascensão de produtos de conveniência com vida útil prolongada, qualidade sensorial e nutritiva. Os vegetais minimamente processados são aqueles que passaram por modificações físicas, ou seja, foram descascados, picados, torneados e ralados, entre outros processos, mas mantidos na forma fresca e, metabolicamente, ativos. Esses produtos são sensíveis à deterioração, bem como podem ser veículos de microorganismos patogênicos ao homem. O objetivo deste estudo foi avaliar a qualidade microbiológica de quatro marcas de folhas de couve manteiga, minimamente processadas, declaradas como higienizadas, comercializadas em supermercados localizados na área metropolitana da cidade de São Paulo. A temperatura de conservação foi mensurada nos locais de venda. As análises microbiológicas realizadas foram contagem padrão de mesófilos aeróbios, leveduras e bolores, teste qualitativo para avaliação da presença de coliformes totais e Escherichia coli. Todos os produtos apresentaram temperaturas acima de $10^{\circ} \mathrm{C}\left(14,3 \pm 1,9^{\circ} \mathrm{C}\right)$, valor este acima da recomendação do fabricante $\left(8^{\circ} \mathrm{C}\right)$ e legislação vigente $\left(<10^{\circ} \mathrm{C}\right.$ por mais que 4 horas). A contagem de mesófilos, leveduras e fungos também apresentou valores acima dos preconizados, $1,0 \times 10^{8} \pm 9,5 \times 10^{7} ; 7,3 \times 10^{7} \pm 8,0 \times 10^{7}$, respectivamente. Foi observada a presença de coliformes totais e Escherichia coli, em todas as amostras, pelo teste qualitativo. Concluiu-se que os produtos analisados, uma vez declarados como higienizados, estavam impróprios para o pronto consumo, sugerindo inadequação no processo de higienização e conservação por parte dos fabricantes e distribuidores, respectivamente. Faz-se necessária a intensificação de ações dos órgãos de fiscalização e saúde. Palavras-chave: higiene dos alimentos; manipulação de alimentos; contaminação de alimentos.

Abstract: The increased demand for ready to eat foods encourages the development of new technologies for food processing. Within this context, fresh foods have attracted consumers, promoting the rise of convenience products with extended shelf life, sensory quality, and nutritive value. The minimally processed vegetables are those that have undergone physical changes, i.e., peeled, chopped, among other processes, but kept fresh and metabolically active. These products are sensitive to deterioration, and can be a vehicle of microorganisms pathogenic to the man. The aim of this study was to evaluate the microbiological quality of four brands of minimally processed kale declared as sanitized, which were commercialized in supermarkets located in the metropolitan area of São Paulo. The storage temperature was measured at the sales location. Microbiological analyzes were performed for

1 Discente do Curso de Nutrição - Universidade Presbiteriana Mackenzie - UPM, Brasil. E-mails: gragesteira@hotmail.com; michi.cm@hotmail.com; litagua@gmail.com; vanessa.l.cardoso@hotmail.com.

2 Doutora em Saúde Pública - Universidade de São Paulo - USP, Brasil e Professora da UPM. Emails: acgmatias@gmail.com; edeli@mackenzie.br. 
mesophilic total, total fungus, and qualitative test for the presence of total coliforms and Escherichia coli. The temperatures of the samples at the purchase time were above that recommended by manufacturers and by current legislation. The total mesophilic counts and total fungal counts showed values above those recommended. The qualitative test found the presence of total coliforms and Escherichia coli in all samples. It was concluded that the products analyzed and declared as sanitary were unfit for ready consumption, suggesting inadequacy in the cleaning process and conservation by the manufacturers and distributors, respectively. Therefore, it is necessary to intensify the actions of organizations of surveillance and heath.

Keywords: food hygiene; food handling; food contamination.

\section{INTRODUÇÃO}

Com o aumento da procura por alimentos prontos para consumo, novas tecnologias vêm sendo desenvolvidas para o processamento de alimentos (LIMA et al., 2003).

Nesse contexto, alimentos frescos estão atraindo a atenção dos consumidores, sendo que o mercado de hortaliças apresenta significativo aumento, desde a década de 90, promovendo a ascensão dos produtos processados frescos. O intuito é proporcionar produtos de conveniência, com vida útil prolongada, qualidade sensorial e nutritiva (OLIVEIRA; VALLE, 2000).

Os vegetais minimamente processados são aqueles que passaram por modificações físicas, ou seja, foram descascados, picados, torneados e ralados, entre outros processos, mas mantidos na forma fresca e metabolicamente ativos (EMPRESA BRASILEIRA DE PESQUISA AGROPECUÁRIA; SERVIÇO BRASILEIRO DE APOIO ÁS MICRO E PEQUENAS EMPRESAS 2007). Comumente, a produção de vegetais frescos minimamente processados é associada a outros tratamentos, como a sanitização.

Hortaliças minimamente processadas apresentam diversos benefícios, tais como: diminuição do tempo de preparo da refeição, aumento da qualidade e padronização, embalagem de armazenamento fácil, necessidade de pouco espaço para armazenamento e diminuição do desperdício (TRESSELER et al., 2009; CENCI, 2000).

No entanto, esses produtos são mais perecíveis, quando comparadas ao mesmo produto intacto, uma vez que são submetidos a processos que causam acentuado estresse físico. Esses danos mecânicos aceleram o metabolismo, consequentemente elevando a taxa respiratória e aumentando a velocidade de deterioração (PICOLI et al., 2010).

As mudanças microbiológicas que ocorrem em hortaliças variam segundo a composição da microflora de cada alimento, que, por sua vez, pode ser alterada por outros fatores, principalmente pelo ambiente, a manipulação, a água disponível, a temperatura, a atmosfera e a acidez (NUNES et al., 2010). Aspecto relevante, em relação ao processamento das hortaliças, é o aumento das superfícies danificadas e a consequente disponibilidade de nutrientes celulares, favorecendo o crescimento microbiano. Somado a isso, o manuseio elevado, durante a preparação destes produtos de conveniência, pode promover contaminação por patógenos cujo habitat é o homem (SIMÕES et al., 2010; CANTWELL, 2000).

Segundo o exposto, este estudo teve como objetivo avaliar a qualidade microbiológica de couve manteiga minimamente processada, comercializada no comércio varejista da região 
central da cidade de São Paulo.

\section{MATERIAL E MÉTODOS}

Foram adquiridas, durante o mês de fevereiro de 2012, em três supermercados localizados na região metropolitana da cidade de São Paulo, quatro amostras, de marcas diferentes, de folhas de couve manteiga minimamente processadas já higienizadas e cortadas. No momento da coleta, foi mensurada a temperatura das amostras, utilizando-se um termômetro digital, do tipo espeto previamente higienizado com álcool 70\% (Incoterm, modelo 6132.08.1.00, RS, BR), que foi posicionado no centro geométrico das amostras. Em seguida, as amostras foram acondicionadas em bolsa térmica com gelo e transportadas até o Laboratório de Bromatologia do Centro de Ciências Biológicas e da Saúde, da Universidade Presbiteriana Mackenzie, onde foram submetidas às análises microbiológicas, no mesmo dia.

Para todos os procedimentos de preparo, contagem, descarte de amostras, bem como métodos analíticos, foram seguidas as recomendações da Instrução Normativa $n^{\circ} 62$, de 26 de agosto de 2003, do Ministério da Agricultura, Pecuária e Abastecimento (BRASIL, 2003).

Para o preparo do experimento, foram pesados $20 \mathrm{~g}$ de cada amostra em uma placa de Petri estéril, seguida de homogeneização com $180 \mathrm{ml}$ de solução salina a $0,85 \%$, em liquidificador previamente esterilizado com álcool $70 \%$, obtendo-se a diluição $10^{-1} \mathrm{e}$, em seguida, procedeu-se a diluição seriada até $10^{-6}$. Todos os experimentos foram realizados em duplicata.

Foi inoculado $1 \mathrm{ml}$ das diluições, por meio de técnica pour plate, em placas contendo Agar Triptona Soja (TSA), para a contagem padrão de mesófilos aeróbios e, em placas contendo Potato Dextrose Agar (PDA), para a contagem padrão de fungos. As placas foram incubadas por 48 horas a $35^{\circ} \mathrm{C}$, e 7 dias a $28^{\circ} \mathrm{C}$, respectivamente. Os resultados foram apresentados como unidades formadoras de colônia por grama de produto (UFC/g).

Foi realizado teste qualitativo para avaliação da presença de coliformes totais, dividido em duas fases: teste presuntivo e confirmativo, adaptado segundo recomendações da Instrução Normativa n 62, do Ministério da Agricultura, Pecuária e Abastecimento (BRASIL, 2003). Para a etapa do teste presuntivo, inocularam-se $10 \mathrm{~mL}$ da diluição $10^{-1}$, em $10 \mathrm{~mL}$ de caldo LST (Lauril Sulfato Triptose), com tubos de Durhan invertidos, e incubou-se a $35^{\circ} \mathrm{C}$, por 48 horas. Imediatamente, após a leitura do teste presuntivo, transferiu-se uma alíquota dos tubos positivos para tubos contendo caldo BLVB (brilho verde brilhante), que foram incubados a $35^{\circ} \mathrm{C}$, por 48 horas, para confirmação da presença de coliformes totais. Os testes foram realizados em duplicata.

Para verificar a presença de Escherichia coli, inoculou-se, por meio da técnica pour plate, $1 \mathrm{ml}$ da diluição $10^{-1} \mathrm{em}$ meio EC, seguida da incubação a $44,5^{\circ} \mathrm{C}$, por 48 horas. Após esse período, na presença de coloração verde metálico confirmou-se a presença de Escherichia coli. 


\section{RESULTADOS E DISCUSSÃO}

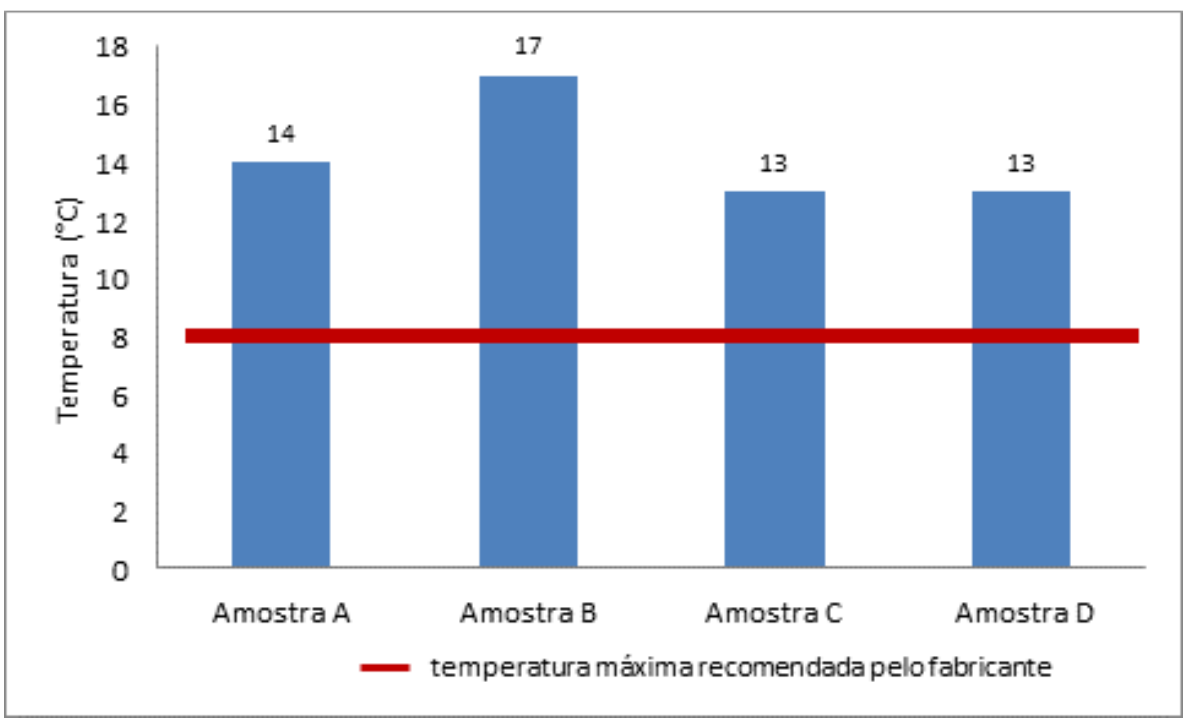

Figura 1 - Distribuição das couves minimamente processadas segundo temperatura no momento da compra e recomendação do fabricante. São Paulo, 2012.

A Portaria no 2619, de 06 de dezembro de 2011, do Município de São Paulo (SÃO PAULO, 2011), preconiza que os alimentos refrigerados devam ser mantidos a temperaturas iguais ou inferiores a $10^{\circ} \mathrm{C}$ ou conforme recomendações do fabricante. Nos rótulos de todas as marcas alvos deste estudo, foi recomendado pelos produtores que as folhas de couve minimamente processadas fossem refrigeradas em temperaturas de 2 a $8^{\circ} \mathrm{C}$. Contudo, conforme a Figura 1, essas recomendações não foram seguidas pelos pontos de venda. No momento da compra, todos os produtos apresentavam temperaturas acima da indicada, as quais ultrapassaram $8^{\circ} \mathrm{C}$ em $62,5 \%\left(13^{\circ} \mathrm{C}\right)$ a $125 \%\left(17^{\circ} \mathrm{C}\right)$. Segundo Simões et al. (2010), o armazenamento de hortalizas minimamente processadas a $10^{\circ} \mathrm{C}$ acelera a atividade enzimática e senescência do vegetal.

Um dos principais problemas associados com hortaliças minimamente processadas é a possibilidade de abuso de temperatura após a embalagem e durante a distribuição, o transporte, o armazenamento, a comercialização, ou antes de serem consumidas. Quando isso ocorre, existe um potencial para proliferação rápida de organismos mesófilos, que apresentam taxa de crescimento pronunciada a $12^{\circ} \mathrm{C}$, resultando na deterioração precoce do produto (THOMAS; O'BEIRNE, 2000).

$\mathrm{Na}$ Tabela 1, são apresentadas a contagem padrão de mesófilos aeróbios e fungos. Segundo Silva Júnior (2008), são consideradas altas contagens de bactérias mesófilas acima de $10^{6} \mathrm{UFC} / \mathrm{g}$ e de bolores e leveduras acima de $5.10^{5} \mathrm{UFC} / \mathrm{g}$. Neste caso todas as amostras apresentaram valores superiores ao adequado. Como possíveis fatores sugeridos para as concentrações observadas, estão a temperatura de armazenamento inadequada nas gôndolas dos supermercados, bem como a possível manipulação inadequada e utensílios mal higienizados. 
Tabela 1 - Distribuição das couves minimamente processadas segundo contagem padrão de mesófilos aeróbios e fungos (UFC/g). São Paulo, 2012

\begin{tabular}{ccc}
\hline Amostras & Mesófilos aeróbios totais & Fungos \\
\hline A & $1,3 \times 10^{7}$ & $1,1 \times 10^{8}$ \\
B & $2,1 \times 10^{8}$ & $1,7 \times 10^{8}$ \\
C & $3,4 \times 10^{7}$ & $1,5 \times 10^{6}$ \\
D & $1,6 \times 10^{8}$ & $1,2 \times 10^{7}$ \\
\hline
\end{tabular}

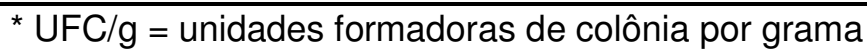

Segundo Tresseler et al. (2009), patógenos mesófilos podem ser introduzidos na área de processamento por vários vetores e podem se estabelecer e multiplicar, particularmente em locais da área de processamento que são difíceis de limpar e sanitizar, tornando-se focos de contaminação.

Em estudo realizado em Porto Alegre - RS, por Silva et al. (2007), verificou-se a qualidade microbiológica de 56 amostras de vegetais minimamente processados, durante 0 período de julho de 2004 a agosto de 2005, e, também, foram constatados valores altos na contagem de microrganismos mesófilos, variando entre $10^{5}$ e $10^{8}$. Recentemente, Pires et al. (2011), em estudo com nove amostras de hortaliças minimamente processadas (batata, mandioca, milho, mandioquinha, alface crespa, alface americana, rúcula, agrião e espinafre), adquiridas em um supermercado de São Paulo, também encontraram-se valores superiores para essa classe de microrganismos, entre $10^{6}$ a $10^{8} \mathrm{UFC} / \mathrm{g}$.

Em relação à contagem de bolores e leveduras, Bruno et al. (2005), também, avaliaram a qualidade microbiológica de 30 amostras de hortaliças, tubérculos e frutas minimamente processadas (legumes crus, mandioca, cenoura e repolho ralados, abacaxi, mamão e salada de frutas, sendo cinco amostras de cada produto), comercializadas em supermercados de Fortaleza - CE, no período de janeiro a maio de 2004, e foi verificada a presença de valores de $10^{2}$ a $10^{6} \mathrm{UFC} / \mathrm{g}$ de bolores e leveduras nas hortaliças minimamente processadas analisadas, indicando segundo os autores, a prática de inadequadas condições de higiene no processamento.

Todas as amostras analisadas nesse estudo acusaram presença de coliformes totais e Escherichia coli, pelo teste qualitativo. A presença de coliformes termotolerantes nos alimentos é indicadora de possível contaminação fecal, ou seja, de condições higiênicosanitárias não satisfatórias (MARQUES; SANTOS; PICCOLI, 2007).

Já, a presença de coliformes termotolerantes, caracterizada pela evidência de Escherichia coli nas amostras, pode significar que, em algum momento do processo produtivo, seja na preparação dos alimentos, na higienização de utensílios e equipamentos, houve a contaminação pelo manipulador. Também, pode-se citar a falha na higienização dos alimentos ou uso de água contaminada (MENDES; SANTOS; OLIVEIRA, 2011).

Para a manutenção da qualidade dos produtos minimamente processados, com redução da carga microbiana patogênica a níveis aceitáveis, bem como aumento da vida útil do produto, é essencial que a etapa de sanitização seja bem realizada (OLIVEIRA; VALE, 2000). 
Frequência semelhante de presença de Escherichia coli foi observada por Pereira e Hoffmann (2011), em São José do Rio Preto, SP, onde 90\% das amostras de vegetais minimamente processados estavam contaminadas.

\section{CONCLUSÃO}

Embora os produtos apresentassem a declaração de higienizados ou sanitizados nos rótulos, eles não estavam adequados para o pronto consumo. As temperaturas de armazenamento estavam acima do recomendado pelos fabricantes, foi observada uma elevada contagem de mesófilos totais e fungos, bem como a presença de termotolerantes e Escherichia coli em todas as amostras. Esses resultados sugerem falhas na sanitização, armazenamento e distribuição desses produtos.

Sugere-se a intensificação de ações de fiscalização e orientação pelos órgãos de controle e saúde, com foco na avaliação e implementação de boas práticas de produção e comercialização de produtos minimamente processados.

\section{REFERÊNCIAS}

BRASIL. Ministério da Agricultura, Pecuária e Abastecimento. Instrução Normativa no 62, de 26 de agosto de 2003. Brasília: DOU, 18/09/2003.

BRUNO, L. M. et al. Avaliação microbiológica de hortaliças e frutas minimamente processadas comercializadas em Fortaleza (CE). Boletim do Centro de Pesquisas e Processamento de Alimentos, Curitiba, v.23, n.1, pp.75-84, 2005.

CANTWELL, M. Preparation and quality of fresh-cut produce. In: ENCONTRO NACIONAL SOBRE PROCESSAMENTO MÍNIMO DE FRUTAS E HORTALIÇAS. 2, 2000, Viçosa. Palestras... Viçosa: UFV, 2000. 110p.

CENCI, S. A. Pesquisa em processamento mínimo de hortaliças no Brasil. In: ENCONTRO NACIONAL SOBRE PROCESSAMENTO MÍNIMO DE FRUTAS E HORTALIÇAS. 2, 2000, Viçosa, MG. Anais... Viçosa: UFV, 2000. 110p.

EMPRESA BRASILEIRA DE PESQUISA AGROPECUÁRIA - EMBRAPA; SERVIÇO BRASILEIRO DE APOIO ÁS MICRO E PEQUENAS EMPRESAS - SEBRAE. Manual de processamento de frutas e hortaliças. Brasília: Embrapa Hortaliças, 2007. 531p.

LIMA, K. S. et al. Cenouras minimamente processadas em embalagens com atmosfera modificadas e tratadas com radiação gama: avaliação microbiológica, físico-química e química. Ciência e Tecnologia de Alimentos, v. 23, n. 2, pp. 240-250, 2003.

MARQUES, S. C.; SANTOS A. L.; PICCOLI, R. H. Pesquisa de Staphylococcus coagulase positiva e coliforme termotolerante em mãos de manipuladores em uma feira de produtos caseiros e artesanais no município de Lavras, MG. Revista Higiene Alimentar, v. 21, n. 155, pp. 23-26. 2007. 
MENDES, C. M.; SANTOS, K. C.; OLIVEIRA, N. L. C. Pesquisa de coliformes totais e termotolerantes em alimentos minimamente processados, comercializados na cidade de Uberlândia, MG. Revista Higiene Alimentar, São Paulo, v. 25, n. 198/199, pp. 58-61, jul./ago., 2011.

NUNES, E. E. et al. Efeito de diferentes temperaturas na qualidade de mandioquinha-salsa minimamente processada. Horticultura Brasileira, Brasília, v. 28, n. 3, pp. 311-315, 2010.

OLIVEIRA, E. C. M.; VALLE, R. H. P. Aspectos microbiológicos dos produtos hortícolas minimamente processados. Revista Higiene Alimentar, São Paulo, v. 11, n. 78/79, pp. 50$54,2000$.

PEREIRA, A. P. M.; HOFFMANN, F. L. Qualidade microbiológica de vegetais minimamente processados. Revista Higiene Alimentar, São Paulo, v. 25, n. 196/197, pp. 60-63, maio/jun. 2011.

PICOLI, A. A. et al. Avaliação de biorreguladores no metabolismo secundário de beterrabas inteiras e minimamente processadas. Bragantia, Campinas, v. 69, n. 4, pp. 983-988, 2010.

PIRES K. R. et al. Qualidade microbiológica de vegetais minimamente processados comercializados na cidade de São Paulo. Revista Higiene Alimentar, v. 25, n. 200/201, pp. 100-104, set./out., 2011.

SÃO PAULO (Município). Secretaria Municipal de Saúde. Portaria no2.619 de 06 de dezembro de 2011 - Regulamento técnico de boas práticas na produção de alimentos. Disponível

em: $<$ http://www.prefeitura.sp.gov.br/cidade/secretarias/upload/chamadas/portaria 2619132369 6514.pdf>. Acesso em: 26 jul. 2012.

SILVA, S. R. P. et al. Microbiological quality of minimally processed vegetables sold in Porto Alegre, Brazil. Brazilian Journal of Microbiology, São Paulo, v. 38, n. 4, pp. 594-598, nov., 2007.

SILVA JUNIOR, E. A. Manual de controle higiênico-sanitário em serviços de alimentação. 6. ed. São Paulo: Varela, 2008. 625p.

SIMÕES, A. N. et al. Effect in the quality of intact and minimally processed leaves of collard greens stored at different temperatures. Horticultura Brasileira, Brasília, v. 28, n. 1, pp. 8186, 2010.

THOMAS, C.; O'BEIRNE, D. Evaluation of the impact of short-term temperature abuse on the microbiology and shelf life of a model ready-to-use vegetable combination product. International Journal of Food Microbiology, v. 59, pp. 47-57, 2000.

TRESSELER, J. F. M. et al. Avaliação da qualidade microbiológica de hortaliças minimamente processadas. Ciência Agrotecnologia, Lavras, v. 33, edição especial, pp. 1722-1727, 2009. 